clinics: to detect first-episode psychosis (FEP) mistakenly identified as a prodrome.

Methods: A clinical audit was conducted of referrals to a UHR service, the Personal Assessment and Crisis Evaluation (PACE) clinic, over a 12-month period (April 2005 to March 2006) to establish the proportion who were psychotic on referral.

Results: The PACE clinic received 149 referrals over the period examined. About $11.4 \%$ of the total number of referrals and $12.6 \%$ of those who attended a first appointment were psychotic on referral.

Conclusions: These figures indicate that a substantial proportion of individuals thought to be prodromal are in fact suffering FEP. It is suggested that by detecting these individuals and commencing treatment, UHR clinics minimize duration of untreated psychosis for patients with FEP mistaken as prodromal.

\section{Obsessive compulsive disorder: time to decide on a new model?}

\author{
A Olley', G Malhi ${ }^{2,3,4}$, P Sachdev ${ }^{1,5}$ \\ 'School of Psychiatry, University of New South Wales; ${ }^{2}$ Academic Discipline of \\ Psychological Medicine, Northern Clinical School, The University of Sydney; ${ }^{3}$ The \\ Black Dog Institute, Sydney, Australia; 4Prince of Wales Medical Research Institute, \\ Sydney, Australia; and ${ }^{5}$ Neuropsychiatric Institute, Prince of Wales Hospital, \\ Sydney, Australia
}

Background: Obsessive compulsive disorder (OCD) is a chronic and debilitating disorder characterized by chronic doubt and indecision; yet, decision making as a cognitive construct has received little attention in either the neuropsychological or the neuroimaging studies of OCD. Indeed, the neuropsychological profile of OCD remains poorly defined and further investigations are needed. To date, most studies have been exploratory in nature and not based on theoretical frameworks.

Methods: A selective review of memory and executive functioning in OCD was conducted using the MEDLINE database, with particular focus on decision making. In addition, the neurobiology of both OCD and decision making was reviewed.

Results: OCD, although once believed to be a disorder of memory, is now viewed primarily as a disorder of executive functioning, a view which is strongly supported by findings in neuroimaging. Decision making, although little studied, is emerging as a potential area of dysfunction that warrants further investigation.

Conclusions: Given the incapacity that results from chronic doubt and indecision in the clinical population, it is surprising that decision making has not received greater consideration in conceptualizing this disorder. We posit a tentative model of OCD that provides a unique perspective of OCD within a decision-making framework. To examine the utility of this model, we propose a multimodal study incorporating functional neuroimaging and neuropsychological assessment in an attempt to dissect the individual stages of decision making in OCD. Understanding the neuroanatomical substrates will lead to the development of new physical and psychological treatments.

\section{They never forget: recall of traumatic memories of Vietnam}

\section{B 0'Toole', K Pierse', J Cockburn², S Catts ${ }^{3}$ \\ 'ANZAC Research Institute; ' University of Newcastle; and ${ }^{3} U$ niversity of Queensland, Brisbane, Australia}

Background: The establishment of a diagnosis of post-traumatic stress disorder (PTSD) requires first that the person has actually experienced a traumatic event. The second wave of the Australian Vietnam Veterans Health Study allowed us to observe the consistency of reports of war trauma 30 or more years after the trauma and 15 years after their initial recounting.

Methods: Interviews were conducted in 2005-2006 with a cohort of randomly selected Australian Army Vietnam veterans who were first assessed using standardized PTSD diagnostic assessments during 19911993. A standardized scale was used on both occasions to measure war trauma exposure and to act as a cue to eliciting recalled and reported events that would qualify under the DSM ' $A$ ' criterion. One or more distressing events were elicited and transcribed for a qualitative analysis of the war stories offered in 2005-2006 and 1991-1993.

Results: Time 1 to time 2 correlations between each of the 21 items in the combat scale were highly positive, as was the total. The factor structures of the two administrations were very similar, as were item-total correlations and Cronbach's $\alpha$. Qualitative analysis of stories showed that almost identical stories were told on the two occasions when nominating the 'worst' events of Vietnam. However, some of the details emerged over several hours of interviewing, which indicates that veterans may not be as open to disclosing their war histories in short encounters.

Conclusion: Soldiers' memory for war-related trauma is very stable, even 40 years after war's end.

\section{The prevalence of PTSD in Australian Vietnam Veterans 35 years after war's end: preliminary results of a cohort study}

\section{B O'Toole', K Pierse', S Outram², J Cockburn², S Catts ${ }^{3}$}

'ANZAC Research Institute; ${ }^{2}$ University of Newcastle; and ${ }^{3}$ University of Queensland, Brisbane, Australia 